\title{
Effect of Collaterals on Clinical Presentation, Baseline Imaging, Complications, and Outcome in Acute Stroke
}

\author{
E.M. Fanou, (D). Knight, R.I. Aviv, S.-P. Hojjat, S.P. Symons, L. Zhang, and (D) M. Wintermark
}

\begin{abstract}
BACKGROUND AND PURPOSE: Good CTA collaterals independently predict good outcome in acute ischemic stroke. Our aim was to evaluate the role of collateral circulation and its added benefit over CTP-derived total ischemic volume as a predictor of baseline NIHSS score, total ischemic volume, hemorrhagic transformation, final infarct size, and a modified Rankin Scale score $>2$.
\end{abstract}

MATERIALS AND METHODS: This was a retrospective study of 395 patients with stroke dichotomized by recanalization (recanalization positive/recanalization negative) and collateral status. Clot burden score was quantified on baseline CTA. Total ischemic volumes were derived from thresholded CTP maps. Final infarct size was assessed on follow-up CT/MRI. We performed uni-/multivariate analyses for each outcome, adjusting for rtPA status, using general linear (continuous variables) and logistic (binary variables) regression. Model comparison with collateral score and total ischemic volume was performed using the $F$ or likelihood ratio test.

RESULTS: Collateral presence independently and inversely predicted all outcomes except hemorrhagic transformation in patients who were recanalization negative and $\mathrm{mRS}>2$ in patients who were recanalization positive. The greatest collateral benefit occurred in patients who were recanalization negative, contributing $16.5 \%$ and $19.2 \%$ of the variability for final infarct size and $\mathrm{mRS}>2$. The collateral score model is superior to the total ischemic volume for $\mathrm{mRS}>2$ prediction, but a combination of total ischemic volume and collateral score is superior for mRS $>2$ and final infarct prediction ( $24 \%$ and $28 \%$ variability, respectively). In patients who were recanalization positive, a model including collateral score and total ischemic volume was superior to that of total ischemic volume for hemorrhagic transformation and final infarct prediction but was muted compared with patients who were recanalization negative $(11.3 \%$ and $16.9 \%$ variability).

CONCLUSIONS: Collateral circulation is an independent predictor of all outcomes, but the magnitude of significance varies, greater in patients who were recanalization negative versus recanalization positive. Total ischemic volume assessment is complementary to collateral score in many cases.

ABBREVIATIONS: $C B S=$ clot burden score; $\mathrm{CS}=$ collateral score; $\mathrm{HT}=$ hemorrhagic transformation; $\mathrm{R}+=$ recanalization positive; $\mathrm{R}-=$ recanalization negative; TIV = total ischemic volume; bNIHSS, baseline NIHSS score

p n the setting of acute ischemic stroke, revascularization therapies are administered with the intent of salvaging ischemic penumbra by restoring antegrade flow. ${ }^{1}$ Even though conventional angiography is considered the gold standard for collateral circu-

Received January 5, 2015; accepted after revision May 14

From the Division of Neuroradiology (E.M.F., J.K., R.I.A., S.-P.H., S.P.S., L.Z.), Department of Medical Imaging, University of Toronto and Sunnybrook Health Sciences Centre, Toronto, Ontario, Canada; and Division of Neuroradiology (M.W.), Department of Radiology, Stanford University, California.

Please address correspondence to Richard I. Aviv, MD, FRCR, Sunnybrook Health Sciences Centre, 2075 Bayview Ave, Toronto, Ontario, Canada M4N 3M5; e-mail: richard.aviv@sunnybrook.ca

= Indicates article with supplemental on-line tables.

http://dx.doi.org/10.3174/ajnr.A4453 lation assessment, CT angiography is increasingly used in triaging patients with acute stroke. ${ }^{2}$

Growing evidence underscores the importance of the collateral circulation in maintaining the penumbra and predicting radiological and clinical response to revascularization., ${ }^{3,4}$ Good CTA collaterals independently predict good outcome in acute ischemic stroke ${ }^{1,2}$ and correlate with smaller admission infarct size. ${ }^{5}$ CTA collateral scoring demonstrates good interrater reliability $^{2,6-8}$; is widely available, including after-hours; and has the advantage of not requiring advanced postprocessing, which is subject to a host of technical differences. ${ }^{9}$ The best means of accurate collateral assessment is debated ${ }^{1,10-13}$; however, irrespective of the method of assessment, collateral status significantly predicts clinical outcome and risk of infarct. ${ }^{2,14,15}$ Limitations of collateral evaluation are that vessel opacification is time- and ac- 
quisition speed-dependent, indicating the need for time-invariant CTA imaging. ${ }^{10,11}$ Additionally, the tissue perfusion status is not directly imaged in contradistinction to CT perfusion, in which penumbral prediction is well-studied. ${ }^{16}$

A recent study suggested that a good clinical outcome could only be achieved in the presence of recanalization and good-tointermediate collateral status. No effect was seen in patients without recanalization. Furthermore, the effect of other comorbid clinical (blood pressure, glycemic status, presence of vascular risk factors, and so forth) or radiological features (clot burden score [CBS], clot location, hemorrhagic transformation [HT]) was not considered in outcome determination. ${ }^{13}$ The relationship of collateral status and these other imaging and clinical stroke predictors, independent of recanalization status, for major outcomes is also not well-established in large acute stroke populations. Emphasis on collateral status has increased due to its recent inclusion in patient selection for endovascular treatment ${ }^{17}$; however, the added predictive value of collateral score (CS) over perfusion imaging assessment of total ischemia is not well-studied. We hypothesized that for a given recanalization status in the absence of perfusion availability, collateral determination significantly predicts baseline stroke severity (quantified by the baseline National Institutes of Health Stroke Scale score [bNIHSS]) and clinical (hemorrhagic transformation, 90-day modified Rankin Scale score of $>2$ ) and radiological outcomes (final infarct volume). In the present study, we also sought to quantify the added value of a CS over CTP-estimated total ischemic volume (TIV). The added contribution was assessed independent of recanalization status and accounted for additional important clinical and imaging covariates in multivariate models.

\section{MATERIALS AND METHODS Study Design and Patient Cohort}

The study and medical chart review were approved by the local research ethics board. Consent was obtained from all patients or legal guardians for participation. We performed a retrospective review of 395 consecutive patients (from a prospectively maintained stroke data base) presenting between 2009 and 2013 with a confirmed anterior circulation stroke diagnosed and treated with IV rtPA by a stroke neurologist within 4.5 hours of onset. Included patients demonstrated a confirmed vessel occlusion on admission stroke work-up, including noncontrast CT, CT angiogram, and CT perfusion. Recanalization was determined on CTA performed at $\leq 24$ hours, while follow-up MR imaging or CT at 5-7 days was used for final infarct assessment. Clinical history, laboratory results, demographics, stroke risk factors, and NIHSS score were collected for all patients by medical chart review. Time to CT was calculated as the time interval between symptom onset (last seen normal for patients with nonwitnessed symptom onset) and the time to arrival in the emergency department CT scanner. A follow-up modified Rankin Scale score was obtained in the stroke prevention clinic at 90 days, with a score of $>2$ defining a poor outcome.

\section{Imaging Acquisition Protocol and Image Postprocessing}

CT stroke protocol was performed on a 64-detector row CT scanner (LightSpeed VCT; GE Healthcare, Milwaukee, Wiscon- sin). Pre- and postcontrast head CT parameters were as follows: $120 \mathrm{kV}$ (peak); $300 \mathrm{~mA}$; rotation, 1 second; section thickness, 5 $\mathrm{mm}$. Baseline and 24-hour CT angiogram parameters were as follows: $0.7-\mathrm{mL} / \mathrm{kg}$ of iodinated contrast agent up to a maximum of $90 \mathrm{~mL}$ (iohexol, Omnipaque $300 \mathrm{mg}$ iodine/mL; GE Healthcare, Piscataway, New Jersey); 5- to 10-second delay; $120 \mathrm{kVp} ; 270$ $\mathrm{mA}$; rotation, 1 second; 1.25 -mm-thick sections; table speed, $20.62 \mathrm{~mm}$ per rotation. Biphasic CT perfusion protocol from the basal ganglia to the lateral ventricles was the following: $80 \mathrm{kVp}$; $150 \mathrm{~mA}$; collimation, $8 \times 5 \mathrm{~mm}$; rotation, 1 second for 45 seconds followed by 6 further acquisitions 15 seconds apart for a total of 135 seconds. Iodinated contrast agent of $0.5 \mathrm{~mL} / \mathrm{kg}$ (maximum, $50 \mathrm{~mL}$ ) at $4 \mathrm{~mL} / \mathrm{s}$ was administered 5 seconds before start of the sequence. Cerebral blood flow, cerebral blood volume, and mean transit time were calculated as previously described by using a delay-corrected algorithm commercially available on CT Perfusion 4 (GE Healthcare). ${ }^{18}$

\section{Image Analysis}

All baseline and follow-up imaging assessments were performed separately by the same neuroradiologist (R.I.A., 10 years of experience) blinded to imaging and clinical outcomes. Baseline imaging features included ASPECTS, clot location, clot burden score, and CS. The occlusion site was considered "proximal" if involving the ICA and proximal M1 MCA segment, whereas clot distal to this was considered "distal." Recanalization status was graded on follow-up CTA by evaluating the restoration of patency of the previously occluded vessel by using the adapted Thrombolysis in Myocardial Infarction score, in which a score of $\geq 2$ was considered recanalizationpositive $(\mathrm{R}+) .{ }^{19}$ Collateral flow was graded on a $0-3$ scale. ${ }^{15}$ Scores of 0 and 1 ( $<50 \%$ of ischemic territory demonstrate collaterals) were considered poor collateral status, whereas scores of $\geq 2$ were assigned a good collateral status. The CBS is a scoring system that defines anterior circulation thrombus extent scored on a scale of $0-10$ by using CTA. Two points are subtracted for thrombus in each of the supraclinoid ICAs and the proximal and distal half of the MCA trunk. One point is subtracted for thrombus in each of the infraclinoid ICAs or anterior cerebral artery and each affected M2 MCA branch (to a maximum of 2). A score of 10 connotes clot absence, while zero represents complete multisegment vessel occlusion. ${ }^{15}$

CT perfusion source images were normalized to Montreal Neurological Institute space and segmented into gray and white matter by using SPM8 software (http://www.fil.ion.ucl.ac.uk/ $\mathrm{spm} / \mathrm{software} / \mathrm{spm} 8$ ). Within the ischemic hemisphere, volumes of core, penumbra proceeding to infarction and benign oligemia were calculated by using previously published institution-validated thresholds for GM and WM. ${ }^{20}$ Absolute CBF (milliliters/ $100 \mathrm{~g} / \mathrm{min}$ ) defined the GM/WM core and GM/WM benign oligemia was defined as $\leq 13.8 / 9.8$ and $\geq 21.4 / 14.1$, respectively. GM/WM penumbra proceeding to infarction was represented by $>13.8$ to $<21.4$ / $>9.8$ to $<14.1$, respectively, while GM/WM benign oligemia was $>21.4 / 14.1$, respectively. ${ }^{20} \mathrm{TIV}$ represents the sum of all thresholded ischemic regions above. Final infarct was traced on follow-up imaging, with either NCCT if clearly delineated or FLAIR MR imaging by using Medical Image Processing, 
Analysis, and Visualization (Version 7.0.2; National Institutes of Health, Bethesda, Maryland).

\section{Statistical Analyses}

Continuous scaled demographic data were expressed as mean \pm $\mathrm{SD}$, whereas discrete data were expressed as median and interquartile range. Frequency data were expressed as proportions. Natural log-transformation was applied if appropriate to some continuous variables to normalize their distribution. Patients were divided into 4 groups according to recanalization and collateral status; groups A and B were recanalization-negative $(\mathrm{R}-$ ) and collateral-negative and -positive, respectively, whereas groups $\mathrm{C}$ and $\mathrm{D}$ were $\mathrm{R}+$ and collateral-negative and -positive, respectively. A Bonferroni-adjusted $P$ value $<.0125$ was considered statistically significant after controlling for multiple comparisons and adjusting for rtPA status, by using general linear regression for continuous variables and logistic regression for binary variables. The outcome was each demographic or clinical variable; the independent variable was a categorical variable of "group" with rtPA as a confounding factor. The interaction term "rtPA $\times$ group" was also inserted; however, no significant interaction was demonstrated, suggesting that rtPA treatment effects are not additive to the significant group effects, permitting inclusion of patients treated both with and without rtPA within each group. Uniand multivariate logistic analyses were performed for each group comparison, with the predefined outcomes of bNIHSS, hemorrhagic transformation, final infarct size, and 90-day modified Rankin Scale, after adjusting for rtPA and collateral status as confounding factors. For each independent predictor odds ratio, 95\% CI was calculated. Only factors with $P<.10$ in univariate analysis were advanced into a multivariate analysis with backward stepwise selection. TIV/CS collinearity was tested by using a variance inflation factor for all outcomes.

After establishing no collinearity (variance inflation factor of $<2$ ), the added benefit of CS over CTP-derived TIV was tested. Each of 2 null-nested models was compared by using an F-test or a likelihood ratio test for continuous or binary outcomes. Null models included either collaterals or TIV, while the alternative model included both collaterals and TIV. The likelihood ratio test statistic was determined by -2 log-likelihood for the null model and $+2 \log$-likelihood for the alternative model, assuming a $\chi^{2}$ distribution. Degrees of freedom were the difference between the number of parameters of the alternative model and the null model. SAS (Version 9.2; SAS Institute, Cary, North Carolina) was used for all analyses, and a $P$ value $<.05$ was considered significant.

\section{RESULTS}

The average patient age was $72 \pm 14$ years with 198 (50.1\%) women. Median (interquartile range) ASPECTS and baseline NIHSS score were 7 (6-9) and 14 (7-19), respectively. A clot burden score of $>6$ was present in $45.8 \%$ (181/395) of patients, and $78.9 \%(310 / 395)$ had good collateral status. Occlusion was proximal in 43\% (170/395) and distal in 57\% (225/395). Intravenous recombinant tissue plasminogen activator was administered in $273 / 395(69.1 \%)$ patients, with a median time to treatment of 147 minutes (interquartile range, 125-179 minutes). Recanaliza- tion occurred in 220/395 (55.7\%) patients diagnosed on CTA at a mean time of $24 \pm 6$ hours; $26 / 220$ demonstrated poor collaterals $(11.8 \%)$. Time to scanning was not significantly different between groups and therefore was not considered in multivariate analyses. Poor collaterals were present in 57/175 (32.6\%) patients without recanalization. The median 90-day mRS was 3 (interquartile range, 2-5), with a good outcome in 144/395 (37\%) patients. Hemorrhagic transformation occurred in 174/395 (44.1\%) of patients. Hemorrhagic infarction (HI 1/2) dominated in 133/174 (76.4\%) patients overall, but no difference in hemorrhage type (hemorrhagic infarction versus parenchymal hematoma) was seen between collateral-positive and -negative groups for patients who were $\mathrm{R}+$ and $\mathrm{R}-(P=.67$ and $P=.31$, respectively $)$. The median final infarct size was $38 \mathrm{~cm}^{3}$ (interquartile range, $10-$ $\left.124.3 \mathrm{~cm}^{3}\right)$.

No significant group differences in rtPA use were present on the basis of baseline thresholded ischemic penumbra or core size, though patients receiving rtPA were treated earlier and tended toward higher baseline NIHSS, more proximal clot location, and smaller final infarct size than those receiving supportive medical care. The absence of collaterals was associated with larger total GM/WM ischemic volumes, including infarct core irrespective of recanalization status (On-line Table 1). Significant group interaction with rtPA was present for all outcome measures except hemorrhagic transformation, which just missed significance $(P=.0178)$. All outcome measures were significantly worse in the absence of collaterals, irrespective of recanalization status, with the exception of hemorrhage, which did not meet statistical significance (On-line Table 1). In $\mathrm{R}+$, however, there was no significant difference in dichotomized mRS, though the median mRS score was significantly lower in the presence of collaterals.

\section{Outcome Assessment and Added Value of CS Assessment over CTP}

Baseline Stroke Severity. Poor collateral circulation, greater clot burden (lower CBS), and TIV were significantly related to bNIHSS, irrespective of recanalization status on uni- and multivariate analyses (On-line Tables 2 and 3). Age remained significant on multivariate analysis for patients who were $\mathrm{R}+$ only $(P<$ .0051). If one considered confounders, collateral absence remained significantly related to bNIHSS. There was no significant interaction between CS and other predictive factors. TIV accounted for $33.3 \%$ of bNIHSS severity compared with $27.5 \%$ by using CS only $(27.5 \% ; P=.0019)$. The highest $R^{2}$ was achieved when both CS and CTP-derived TIV were included (36.5\%) (Online Table 3).

Hemorrhagic Transformation. HT risk was not related to rtPA or collateral status in patients who were $\mathrm{R}-(\mathrm{On}$-line Table 2). In patients who were $\mathrm{R}+$ but not $\mathrm{R}-$, collateral status was a significant predictor of HT (On-line Table 2). In multivariate analysis, collateral presence (OR, 0.36 ; 95\% CI, $0.14-0.88$; $P=.0284$ ) was inversely related to HT risk in patients who were $\mathrm{R}-$, while proximal clot location (OR, 2.22; 95\% CI, 1.21-4.12; $P=.0102)$ and hyperglycemia (OR, 3.07; 95\% CI, 1.45-6.70; $P=.0039$ ) were positively related to $\mathrm{HT}$ in patients who were $\mathrm{R}+(\mathrm{On}$-line Table 

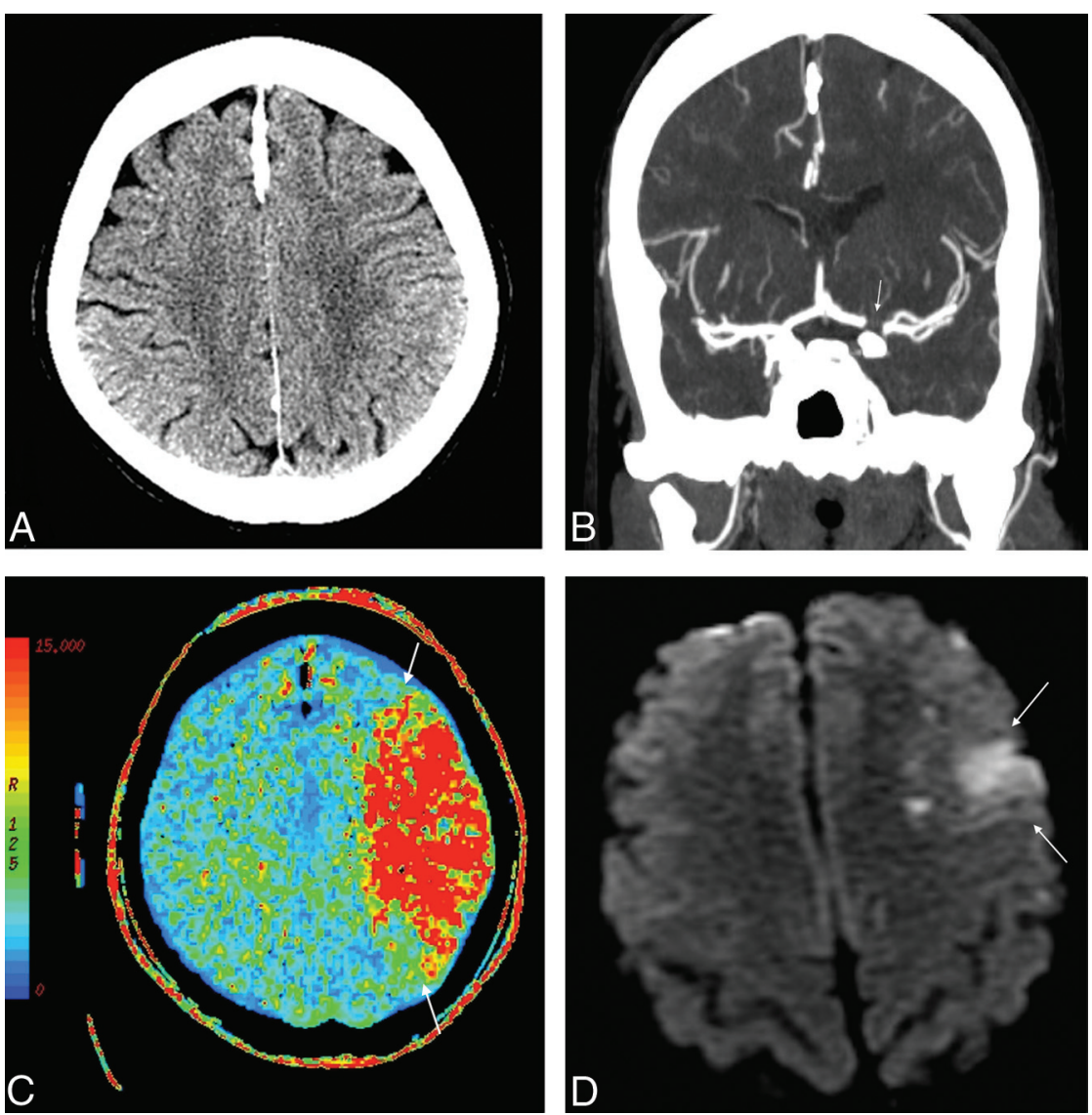

FIG 1. A 57-year-old woman who presented 69 minutes from symptom onset with right-sided acute stroke symptoms. Significant medical history included atrial fibrillation. Her baseline NIHSS score was 11. NCCT shows an ASPECTS of $10(A)$. CTA shows carotid terminus occlusion involving the proximal M1 segment of the left MCA and proximal A1 segment of the left anterior cerebral artery $(C B S=5)$ (arrow) with a collateral score of $3(B)$. The CTP MTT map shows perfusion abnormality involving the left MCA territory ( $C$, arrows). She received rtPA. Follow-up CTA shows nonrecanalization of the occluded vessels (not shown). DWI shows infarct in the left superior frontal region ( $D$, arrows), significantly smaller than the initial perfusion deficit, highlighting the role of collateral circulation in maintaining the penumbra and its association with smaller infarct size. The follow-up mRS score was 2.

3). In contradistinction to CS, TIV was significantly associated with $\mathrm{HT}$ in patients who were $\mathrm{R}-$ but not in those who were $\mathrm{R}+$. No significant model differences were seen in patients who were $\mathrm{R}-$, in whom CS and TIV accounted for between 5.7\% and 7.3\% of HT variability, respectively; however in patients who were $\mathrm{R}+$, CS contributed significantly to a model including TIV, accounting for $11.3 \%$ of HT variability (On-line Table 4 ).

mRS Greater Than 2. The risk of $m R S>2$ was significantly related to collaterals in patients who were $\mathrm{R}-$ but not in those who were $\mathrm{R}+$. Multivariate analysis demonstrated that in patients who were $\mathrm{R}-$, collateral presence was inversely related (OR, 0.15; 95\% CI, $0.04-0.48 ; P=.0037)$ to $\mathrm{mRS}>2$. Older age was associated with poor outcome in both $\mathrm{R}-$ and $\mathrm{R}+$ groups (OR, 1.04, 95\% CI, 1.01-1.07; $P=.0046$ and OR, 1.05; 95\% CI, 1.03-1.08; $P<$ .0001 , respectively.) In patients who were $\mathrm{R}-$, CS added significantly to a model including only TIV and accounted for $24.1 \%$ of mRS $>2$ variability (On-line Table 3 ).

Final Infarct Size. The absence of collaterals was strongly associated with larger infarct size irrespective of recanalization status $(P<.0006$ and $P=.0001)$. In patients who were $\mathrm{R}-$ and $\mathrm{R}+$, collateral absence $(P=.0005, P=.0026)$ and lower CBS $(P=.0028, P=.002)$, respectively, were strongly associated with larger infarct size (On-line Table 2). Patients who were $\mathrm{R}+$ receiving $\mathrm{rtPA}$ $(P=.0276)$ demonstrated smaller infarcts. For patients who were $\mathrm{R}-$, CS contributed little to overall infarct prediction in the presence of TIV assessment, but a model including both CS and TIV accounted for greater variability than CS alone (28\% versus $16.5 \% ; P=.005$, respectively). For patients who were $\mathrm{R}+$, CS significantly improved a model of only TIV and accounted for $16.9 \%$ variability for infarct volume (On-line Table 3).

\section{DISCUSSION}

Our study demonstrates that collaterals are important for all measured outcomes irrespective of recanalization. However, the magnitude of the added benefit of CS over TIV assessment is dependent on recanalization status, and in many cases, CS and TIV are complementary. The greatest benefit of collaterals occurs in the absence of recanalization, in which collaterals contribute to $16.5 \%$ of the variability of final infarct size and $19.2 \%$ of the likelihood of mRS $>2$ but not to the risk of HT. Our results, therefore, extend the findings of a recent study demonstrating effect modification of collaterals in patients who were $\mathrm{R}+$ but not $\mathrm{R}-.{ }^{13}$ Notably, CS adds benefit over TIV for prediction of $\mathrm{mRS}>2$, but a combination of both TIV and CS is superior to CS alone for both $\mathrm{mRS}>2$ and final infarct prediction, accounting for $24 \%$ and $28 \%$ of outcome variability, respectively. In patients who were $\mathrm{R}+$, a model including both CS and TIV was superior to that with TIV alone for prediction of HT and final infarct. The variability associated with these outcomes was more muted compared with patients who were $\mathrm{R}-$ and accounted for $11.3 \%$ and $16.9 \%$ of the model's prediction of HT and final infarct size, respectively, in $\mathrm{R}+$ patients. An inverse association between collateral presence and $\mathrm{mRS}>2$ was demonstrated in patients with $\mathrm{R}+$ and $\mathrm{R}$ - status but was most significant in the absence of recanalization. Most important, the proportion of patients of $\mathrm{mRS}>2$ in patients who were $\mathrm{R}$ - with good collaterals was similar to that of patients who were collateral-negative and $\mathrm{R}+$, reinforcing the importance of both recanalization and collaterals in avoiding futile recanalization..$^{21,22}$

The findings underscore the utility of collateral assessment in predicting outcome irrespective of final recanalization status, consistent with findings of others. ${ }^{2,4}$ Preserved collaterals irrespective of recanalization status were associated with reduced 

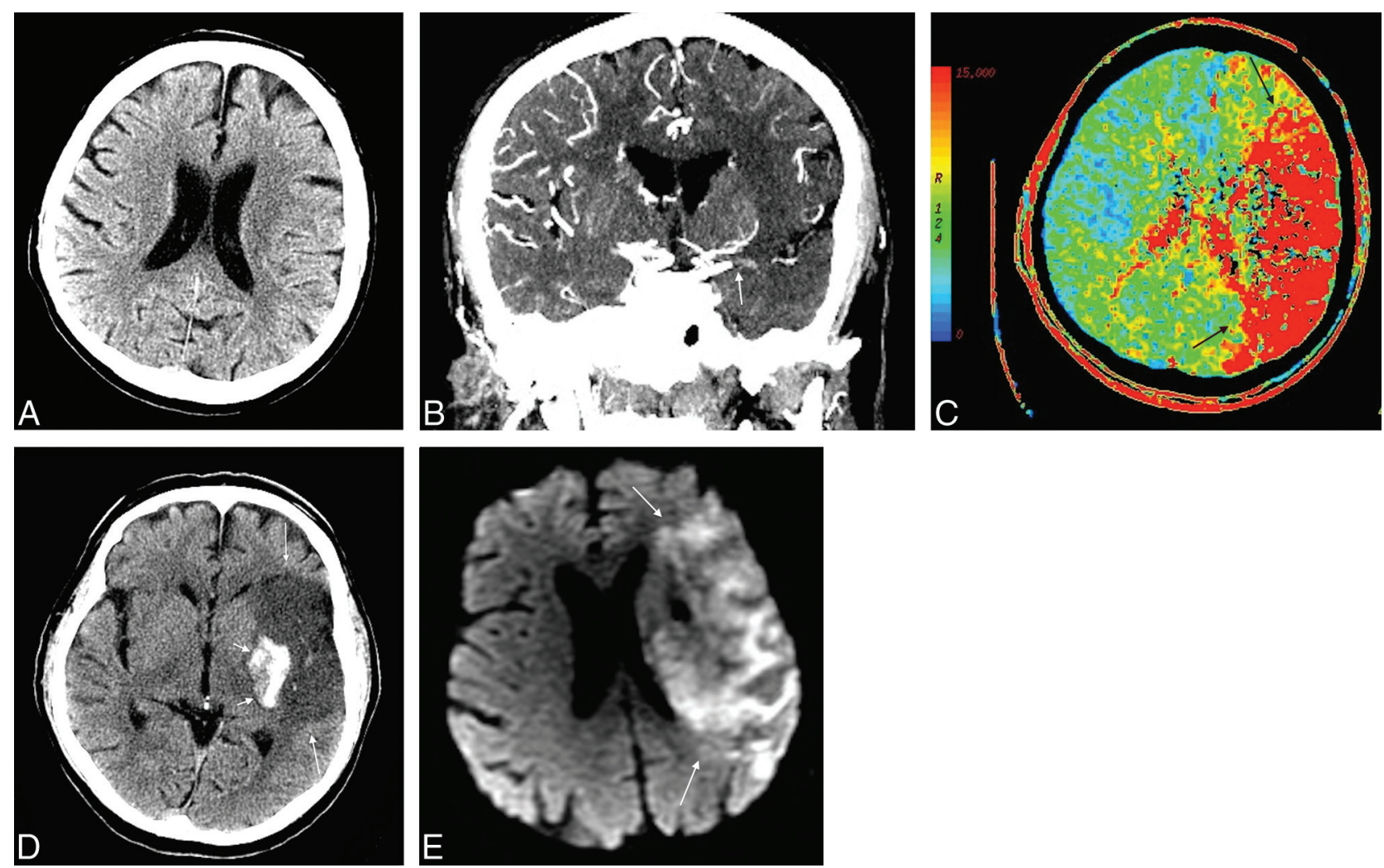

FIG 2. A 69-year-old man who presented 179 minutes from symptom onset with right-sided acute stroke symptoms. Significant medical history included diabetes mellitus, hypertension, high cholesterol, and smoking. His baseline NIHSS score was 25. NCCT shows an ASPECTS of 6 (A). CTA shows occlusion of the M1 segment of the left MCA and proximal M2 segment of the left MCA $(C B S=4)$ (arrows) with a CS of 1 ( $B$ ). The CTP MTT map shows perfusion abnormality involving almost the entire left MCA territory (C, arrows). He received rtPA. Twenty-four-hour CTA shows nonrecanalization of the occluded vessels (not shown), and follow-up DWI on day 5 shows a large infarct in the left MCA territory (E, arrows), similar in size to the initial perfusion deficit. NCCT shows hemorrhagic transformation in the left basal ganglia region ( $D$, small arrows). Follow-up mRS was 4.

baseline ischemic tissue (including baseline infarct core) and smaller final infarct volume, contributing to a better outcome (Fig 1). The findings are consistent with prior studies emphasizing the role of collaterals in preserving penumbral viability by minimizing ischemic injury severity and maintaining tissue perfusion. $^{2,4,23-25}$

In the present series, no significant difference in HT frequency was present in the absence of recanalization irrespective of collateral status. Patients who were $\mathrm{R}+$ without collaterals were more likely to undergo HT than those with collaterals. Although recanalization contributes significantly to better outcome in stroke series, our results emphasize that recanalization in the absence of collaterals may have significant adverse outcomes, such as increased HT following restoration of blood flow to nonviable tissue and damaged vasculature. ${ }^{26}$ This finding is in agreement with IV rtPA and endovascular therapy studies, in which poor collateral status was associated with higher rates of HT. ${ }^{7,23}$ The frequency of poor collaterals in the present series $(11 \%$ and $30 \%$ in patients who were $\mathrm{R}-$ and $\mathrm{R}+$, respectively) is similar to that of other series $^{2,7,24}$; this similarity indicates that most patients with acute ischemic stroke manifest collateral preservation at baseline and are eligible for recanalization therapy.

CBS $<6$ (reflecting larger and usually more proximal clot) was independently associated with greater bNIHSS and infarct volumes, irrespective of recanalization and collateral status. ${ }^{27}$ In- creased clot burden (low CBS) was also associated with a higher risk of HT (Fig 2). Collateral status is critically dependent on the level and magnitude of clot burden. More proximal clot is associated with larger baseline infarct core and penumbral tissue volumes. These appearances are mediated by a combination of reduced/delayed proximal clot lysis, reduced collateral pathway availability, and cerebral hypoperfusion, provoking profound ischemia inconsistent with viable tissue. ${ }^{27-29}$ Indeed Saqqur et $\mathrm{al}^{30}$ demonstrated that patients with more distal clot were twice as likely to have a favorable clinical outcome as patients with more proximal occlusions. Hence, careful evaluation of collateral status should be made in patients with lower CBS before aggressive recanalization strategies.

We considered the role of collaterals in bNIHSS. Clinical deficit in the hyperacute phase reflects the size of both the infarcted and penumbral zones, whereas early NCCT findings reflect only infarcted tissue. An NIHSS/CT mismatch, therefore, represents salvageable brain ${ }^{31}$ and indicates a risk of early neurologic deterioration. ${ }^{32}$ We demonstrated that age, clot location, and collaterals were independently associated with stroke severity (On-line Table 2); younger patients with good collateral circulation and distal clot are more likely to have a good outcome. Hyperglycemia was associated with a higher risk of hemorrhagic transformation in patients who were recanalization-positive. Studies evaluating outcome in patients with diabetes and acute stroke have been contradictory. In one study of patients with diabetes and acute ischemic stroke 
treated with IV-rtPA, baseline glucose and diabetes were independent predictors of intracranial hemorrhage. ${ }^{33}$ However more recent studies have refuted the association between diabetes and intracranial bleed, ${ }^{34}$ rather suggesting that hyperglycemia itself accelerates vascular injury in the ischemic area through biochemical changes in the endothelium and by hampering fibrinolysis. ${ }^{35,36}$

A limitation of the present study in view of recent endovascular study results is that none of the patients in the present series underwent mechanical or intra-arterial thrombolysis. Therefore, results are limited to those receiving IV rtPA only. In the present series, a consistent characterization of recanalization was performed at around 24 hours for all patients, thereby attenuating any systematic bias that may have been introduced. However, this approach would not distinguish between early and late recanalizers. Nevertheless the expected findings of reduced infarct size and better outcome in the context of recanalization support our definition as clinically relevant.

\section{CONCLUSIONS}

Collateral circulation is an independent predictor of bNIHSS, final infarct volume, hemorrhagic transformation, and $\mathrm{mRS}>2$. The extent of significance varies between patients who are recanalization-negative and -positive, with the greatest impact in those without recanalization. TIV assessment is complementary to CS in many cases.

Disclosures: Sean P. Symons_UNRELATED: Payment for Lectures (including service on Speakers Bureaus): Biogen Idec Canada (for MS talks).

\section{REFERENCES}

1. Menon BK, Smith EE, Modi J, et al. Regional leptomeningeal score on $\mathrm{CT}$ angiography predicts clinical and imaging outcomes in patients with acute anterior circulation occlusions. AJNR Am J Neuroradiol 2011;32:1640-45 CrossRef Medline

2. Lima FO, Furie KL, Silva GS, et al. The pattern of leptomeningeal collaterals on CT angiography is a strong predictor of long-term functional outcome in stroke patients with large vessel intracranial occlusion. Stroke 2010;41:2316-22 CrossRef Medline

3. Zaidat OO, Yoo AJ, Khatri P, et al; Cerebral Angiographic Revascularization Grading (CARG) Collaborators, STIR Revascularization working group, STIR Thrombolysis in Cerebral Infarction (TICI) Task Force. Recommendations on angiographic revascularization grading standards for acute ischemic stroke: a consensus statement. Stroke 2013;44:2650-63 CrossRef Medline

4. Bang OY, Saver JL, Kim SJ, et al. Collateral flow predicts response to endovascular therapy for acute ischemic stroke. Stroke 2011;42: 693-99 CrossRef Medline

5. Souza LC, Yoo AJ, Chaudhry ZA, et al. Malignant CTA collateral profile is highly specific for large admission DWI infarct core and poor outcome in acute stroke. AJNR Am J Neuroradiol 2012;33: 1331-36 CrossRef Medline

6. Lee M, Saver JL, Alger JR, et al. Blood-brain barrier permeability derangements in posterior circulation ischemic stroke: frequency and relation to hemorrhagic transformation. J Neurol Sci 2012;313: 142-46 CrossRef Medline

7. Brunner F, Tomandl B, Hanken K, et al. Impact of collateral circulation on early outcome and risk of hemorrhagic complications after systemic thrombolysis. Int J Stroke 2014;9:992-98 CrossRef Medline

8. Shuaib A, Butcher K, Mohammad AA, et al. Collateral blood vessels in acute ischaemic stroke: a potential therapeutic target. Lancet Neurol 2011;10:909-21 CrossRef Medline

9. González RG. Current state of acute stroke imaging. Stroke 2013;44: 3260-64 CrossRef Medline
10. Frölich AM, Wolff SL, Psychogios MN, et al. Time-resolved assessment of collateral flow using 4D CT angiography in large-vessel occlusion stroke. Eur Radiol 2014;24:390-96 CrossRef Medline

11. Smit EJ, Vonken EJ, van Seeters T, et al. Timing-invariant imaging of collateral vessels in acute ischemic stroke. Stroke 2013;44:2194-99 CrossRef Medline

12. Menon BK, O’Brien B, Bivard A, et al. Assessment of leptomeningeal collaterals using dynamic CT angiography in patients with acute ischemic stroke. J Cereb Blood Flow Metab 2013;33:365-71 CrossRef Medline

13. Nambiar V, Sohn SI, Almekhlafi MA, et al. CTA collateral status and response to recanalization in patients with acute ischemic stroke. AJNR Am J Neuroradiol 2014;35:884-90 CrossRef Medline

14. Angermaier A, Langner S, Kirsch M, et al. CT-angiographic collateralization predicts final infarct volume after intra-arterial thrombolysis for acute anterior circulation ischemic stroke. Cerebrovasc Dis 2011;31:177-84 CrossRef Medline

15. Tan IY, Demchuk AM, Hopyan J, et al. CT angiography clot burden score and collateral score: correlation with clinical and radiologic outcomes in acute middle cerebral artery infarct. AJNR Am J Neuroradiol 2009;30:525-31 CrossRef Medline

16. Bivard A, Levi C, Spratt N, et al. Perfusion CT in acute stroke: a comprehensive analysis of infarct and penumbra. Radiology 2013; 267:543-50 CrossRef Medline

17. Goyal M, Demchuk AM, Menon BK, et al; ESCAPE Trial Investigators. Randomized assessment of rapid endovascular treatment of ischemic stroke. N Engl J Med 2015;372:1019-30 CrossRef Medline

18. Eilaghi A, Brooks J, d'Esterre C, et al. Reperfusion is a stronger predictor of good clinical outcome than recanalization in ischemic stroke. Radiology 2013;269:240 -48 CrossRef Medline

19. Tomsick T. TIMI, TIBI, TICI: I came, I saw, I got confused. AJNR Am J Neuroradiol 2007;28:382-84Medline

20. Eilaghi A, d'Esterre CD, Lee TY, et al. Toward patient-tailored perfusion thresholds for prediction of stroke outcome. AJNR Am J Neuroradiol 2014;35:472-77 CrossRef Medline

21. Murphy A, Symons SP, Hopyan J, et al. Factors influencing clinically meaningful recanalization after IV-rtPA in acute ischemic stroke. AJNR Am J Neuroradiol 2013;34:146-52 CrossRef Medline

22. Molina CA. Futile recanalization in mechanical embolectomy trials: a call to improve selection of patients for revascularization. Stroke 2010;41:842-43 CrossRef Medline

23. Bang OY, Saver JL, Kim SJ, et al; UCLA-Samsung Stroke Collaborators. Collateral flow averts hemorrhagic transformation after endovascular therapy for acute ischemic stroke. Stroke 2011;42:2235-39 CrossRef Medline

24. Miteff F, Levi CR, Bateman GA, et al. The independent predictive utility of computed tomography angiographic collateral status in acute ischaemic stroke. Brain 2009;132(pt 8):2231-38 CrossRef Medline

25. Jung S, Gilgen M, Slotboom J, et al. Factors that determine penumbral tissue loss in acute ischaemic stroke. Brain 2012;136(pt 12): 3554-60 CrossRef Medline

26. Campbell BC, Christensen S, Tress BM, et al; EPITHET Investigators. Failure of collateral blood flow is associated with infarct growth in ischemic stroke. J Cereb Blood Flow Metab 2013;33:1168-72 CrossRef Medline

27. Zhu G, Michel P, Aghaebrahim A, et al. Computed tomography workup of patients suspected of acute ischemic stroke: perfusion computed tomography adds value compared with clinical evaluation, noncontrast computed tomography, and computed tomography angiogram in terms of predicting outcome. Stroke 2013;44: 1049-55 CrossRef Medline

28. Gasparotti R, Grassi M, Mardighian D, et al. Perfusion CT in patients with acute ischemic stroke treated with intra-arterial thrombolysis: predictive value of infarct core size on clinical outcome. AJNR Am J Neuroradiol 2009;30:722-27 CrossRef Medline

29. Yoo AJ, Verduzco LA, Schaefer PW, et al. MRI-based selection for 
intra-arterial stroke therapy: value of pretreatment diffusionweighted imaging lesion volume in selecting patients with acute stroke who will benefit from early recanalization. Stroke 2009;40: 2046-54 CrossRef Medline

30. Saqqur M, Uchino K, Demchuk AM, et al; CLOTBUST Investigators. Site of arterial occlusion identified by transcranial Doppler predicts the response to intravenous thrombolysis for stroke. Stroke 2007;38:948-54 CrossRef Medline

31. Kent DM, Hill MD, Ruthazer R, et al. "Clinical-CT mismatch" and the response to systemic thrombolytic therapy in acute ischemic stroke. Stroke 2005;36:1695-99 CrossRef Medline

32. Tei H, Uchiyama S, Usui T. Clinical-diffusion mismatch defined by NIHSS and ASPECTS in non-lacunar anterior circulation infarction. J Neurol 2007;254:340-46 CrossRef Medline
33. Demchuk AM, Morgenstern LB, Krieger DW, et al. Serum glucose level and diabetes predict tissue plasminogen activator-related intracerebral hemorrhage in acute ischemic stroke. Stroke 1999;30: 34-39 CrossRef Medline

34. Reiter M, Teuschl Y, Matz K, et al; Austrian Stroke Unit Registry Collaborators. Diabetes and thrombolysis for acute stroke: a clear benefit for diabetics. Eur J Neurol 2014;21:5-10 CrossRef Medline

35. Ribo M, Molina C, Montaner J, et al. Acute hyperglycemia state is associated with lower tPA-induced recanalization rates in stroke patients. Stroke 2005;36:1705-09 CrossRef Medline

36. Martini SR, Kent TA. Hyperglycemia in acute ischemic stroke: a vascular perspective. J Cereb Blood Flow Metab 2007;27:435-51 CrossRef Medline 